\title{
An In-Depth Analysis of the Various Factors Influencing Industry 4.0 and its Impact on the Overall Performance of the Industrial Sector to Achieve Sustainable Development
}

\author{
https://doi.org/10.3991/ijoe.v17i11.25325 \\ Pritam Kumar \\ Assumption University, Bangkok, Thailand \\ pkumar@msme.au.edu
}

\begin{abstract}
In the last two years, the entire world has been confronted with the emergence of Covid 19 pandemic, which poses several challenges to the entire human race. Covid 19 has been forced to adopt new innovative technology. To survive in this precarious situation; most of the organization has adopted new innovative technology to meet the expectations of the market and the demands of consumers. In this study, the primary goal is to identify the various factors that have an impact on Industry 4.0 while also evaluating the impact of Industry 4.0 on the overall performance of the industrial sector. An exploratory research approach is used by researchers to discover the characteristics and relationships of new research phenomena. This approach is particularly useful when researchers are looking for answers to the questions of what and how they came to be discovered. This study demonstrates that the use of Industry 4.0 technologies will increase the effectiveness and efficiency of organizations and industries. This is beneficial because organizations and industries need to develop and survive in this competitive era. Adoption of smart machine and smart product applications will become more common with the widespread use of information and communication technology for all aspects of business operations and activities.
\end{abstract}

Keywords-industry 4.0, industrial revolution, big data, technology adoption

\section{$1 \quad$ Introduction}

It is known as the Fourth Industrial Revolution [also known as Industry 4.0 [IR4.0]]. It is a current emergence issue that is characterized by the rapid development and growth of innovative technologies that help industries to achieve sustainable development and to survive in this highly competitive market. In the last two years, the entire world has been confronted with the emergence of Covid 19 pandemic, which poses several challenges to the entire human race. Covid 19 has been forced to adopt new innovative technology to survive in this precarious situation; most of the organization has adopted new innovative technology to meet the expectations of the market and the demands of consumers. Industry 4.0, which includes the use of innovative technologies 
in production, marketing, finance, human resource management, inventory management, supply chain management, consumer relationship management, and other activities, is the preferred strategy for most organizations for achieving this goal [14]. The Internet of Things, big data, data prototyping, and analytics are all components of Industry 4.0. The German government was the first to make use of the term "Industry 4.0." Industry 4.0 is defined as the extensive theoretical and practical application of digitalization and innovative technologies to understand and explore fundamental knowledge about the application of technology for sustainable development, as well as improved organizational performance [1]. 5 years after its debut in Germany, the Industry 4.0 idea has spread around the world, and it's also been moved from its primary application field in industrial production towards other engineering and non-engineering disciplines. Only significant use of ICT tools, connection, and the gathering and analysis of real-time data are shared by similar ideas such as Auto 4.0, Logistics 4.0, and Learning 4.0 with the original meaning of Industry 4.0. From the other hand, Industry 4.0 may be viewed as a natural transition of industrial production processes caused by the digitization trend [13].

Every organization is distinct from the others in terms of its structure, size, objective, and other factors; therefore, the success of an organization varies depending on the approaches, experiences, and skills of the organization. As a result of the emergence of technological innovation and the continued development of the industrial context, the global economy as well as industrial practices have changed dramatically in the last few years [5]. Production technology, automated machines, advanced materials, marketing, supply chain, extra manufacturing, and other technologies are all changing at the same time, and manufacturing organizations must adapt. The increased demand for connectivity with all stakeholders, as well as the gathering, collection, and analysis of data, has resulted from Covid 19. Industry 4.0 enhances the capabilities and abilities made possible by the Internet of Things, and it has resulted in a shift away from mechanical technology and toward information-based technology [4]. When information technology organizations improve their connectivity, it becomes possible to develop, implement, and control supply chains, distribution systems, and the overall activities and processes of the organization.

The success of an organization is measured by the growth of the organization or the level of employee satisfaction. One of the most important factors determining organizational success is the organization's ability to achieve its predetermined goals and objectives. Naturally occurring disasters, pandemic situations, and climate change, among other things, are examples of natural environmental factors. In addition to natural environmental factors, other factors such as legal, social, technological, economic, governmental, and political factors (external environment) have an impact on the productivity and performance of an organization. The current Covid 19 pandemic has given rebirth to Industry 4.0, which is already altering the technological, social, political, and natural factors of the external environment in all industrial sectors, including the pharmaceutical industry. As the business environment changes, it is necessary for the market and industry to constantly update and improve their technology and themselves to remain competitive. It ensures the continuity of business operations in stressful situations. This paper focuses on modernizing and advancing industry, as well as on updating business 
processes and activities, to achieve sustainable development. To increase the value of an enterprise and improve performance, new and innovative technologies such as mobile Internet, cyber-physical systems (CPS), wireless sensor networks, big data, cloud computing, data mining, data warehouse, and collaborative robots are being implemented. All these applications offer some benefits to community, institutions, and country [15], to improve performance and increase efficiency by providing employee training, lowering costs, and saving time, among other things. All these possibilities could assist traditional manufacturing companies in adopting, implementing, and controlling Industry 4.0 technologies with the least amount of expense and risk [9]. The Fourth Industrial Revolution [Industry 4.0] presents a slew of opportunities for the manufacturing industry. It includes the development of new skills, the acquisition of realtime data access, and the achievement of sustainable development through the implementation of Industry 4.0. Industry 4.0 provides real-time data for analysis and decision-making, allowing for faster decision-making. A new opportunity has presented itself to the business organization to eliminate or reduce the difficult challenges associated with the implementation of Industry 4.0 systems.

\section{Literature review}

There are various published research works, articles in peer-reviewed journals, and magazine articles included in the literature review. Achievement with industry 4.0 is highly reliant on a set of skills that encourages continuous collaboration between humans and robots $[3,17]$. This review contributes to the development of the theoretical foundation for the current study, which explains the relationship between Industry 4.0 and the performance of an organization. According to the findings of this study, most authors and researchers are attempting to determine the factors and challenges that influence the adoption and implementation of Industry 4.0 solutions.

Nine different criteria [6] were determined that influence the industry 4.0 trend and analyzed the impacts on different organization strengths and weaknesses. Using the structural equation model (SEM) to determine results, this study looked at nine criteria, including information technology architecture, products and services of businesses, business models; market and customer access; value chains and processes; compliance; risk; security; leadership; strategy; culture; organization and corporate culture; management; and the Internet of Things. There were other requirements such as data integration for analysis, data processing and protection for the team, team expertise, and value reliability. All these factors contributed significantly to the success in the adoption and implementation of Industry 4.0. All this research determined that Industry 4.0 had a positive impact.

Focusing on the driving force behind upgrading and promoting the quality of manufacturing in China's manufacturing industry, the impact of Industry 4.0 on the organization performance is very important [8]. Textual mining is used to sort data and information for this aim by the researchers. As a sample, 460 companies that were implementing the industry 4.0 strategy were selected for this study. Industry 4.0 driving forces were investigated using a probit model during the research phase, which was 
conducted at the time of adoption and implementation. In the study, the findings revealed that private and large companies were more motivated to adopt and implement the industry 4.0 strategy, and that government subsidies had no significant impact on the decision-making of firms when it came to the adoption and implementation of the industry 4.0 strategy. The implementation of Industry 4.0 can significantly improve a company's innovation activities, financial performance, stock returns but it has had no significant impact on the efficiency of the supply chain. The implementation of Industry 4.0 had a positive impact on the information transparency of the company.

Industry 4.0, as defined by computer advancements, provides deep insights into smart manufacturing [4], the adoption of new technology and digitalization has become a more common component in manufacturers, as it makes business processes easier while also making their products and services more intelligent. Several findings from this study are applicable to increased responsiveness and efficiency in a manufacturing process in a manufacturing environment. According to the findings of this study, every business organization is subjected to constant change and uncertainty in the workplace environment. According to the findings of this study, several factors must come together for Industry 4.0 to be successful. These factors include appropriate investment in skills and training, economic and political factors, as well as the availability of advanced tools to facilitate the adoption and implementation of Industry 4.0.

Industry 4.0 technology has attracted popularity since they can give numerous advantages such as enhanced effectiveness, reduced costs, and higher revenues. The study's key success factors, the understanding of parameters [2]. To understand and identify the performance outcomes and critical success factors of industry 4.0 technologies, the researcher reviewed and identified various published literature. The relationships between critical success factors and performance outcomes were discovered by the researcher using regression analysis. According to the findings of the study, data governance was the most important factor that had a direct impact on the performance outcomes, which included responsiveness, product, operational, and economic outcomes, among other things. Out of the four performance outcomes, operational, product, and economic performance are all affected by legal considerations. Furthermore, the findings of this study demonstrate that teamwork and collaboration have an impact on the responsiveness and operational performance of an organization. Furthermore, this study provides a fundamental understanding of the factors that were critical in achieving the performance outcomes of industry 4.0 technologies in the automotive manufacturing industry. The findings may also be useful in assisting automotive manufacturing companies in formulating decisions regarding the various strategies that will be required for the adoption and implementation of Industry 4.0 technologies.

To better understand various disruptive applications in managing quality systems at intelligent manufacturing by improving manufacturing processes and ensuring the long-term development of organizations [10], researcher conducted research. A survey method was used by the researcher to collect data and information from production managers of manufacturing companies that had been specifically chosen by the researcher. The researcher selected production managers and supervisors as respondents to collect data related to quality assurance, calibration, and monitoring of the manufacturing process, as well as issues and challenges related to quality control systems in the 
manufacturing industry. The data in this study was analyzed using the U Test, the Mann-Whitney Test, and the Pearson's Test T-test. This research identified advanced analytical applications, tools, and technologies that can be used to improve the manufacturing process and the quality of the finished product. Organizations develop organizational goals that are attainable with the assistance of strategic management techniques [11]. Today, Industry 4.0 is forcing businesses to adapt to new realities. This has created difficulties in formulating and implementing effective strategies to meet the demands of the new business environment. In this study, some strategic planning was undertaken with the goal of minimizing changes while also assisting in the identification of various critical factors that influenced the success of an organization, strategic management, and the implementation of Industry 4.0.

Various factors [5], have had an impact on the implementation of Industry 4.0 technologies in Peruvian micro, small, and medium-sized businesses. According to the findings of this study, it had four factors as a sample, which respond to the main question of this research study, namely, a lack of financial investment, a lack of skilled workers, a lack of advanced technology, and a lack of management vision. The researcher designed a semi-structured interview schedule to collect primary data from the selected respondent. The sample size for the manufacturing sector was 49 businesses, which the researcher used to collect data on the industry. A survey of business managers was conducted by the researcher to gather primary data, and all of the data was evaluated using a multi-criteria decision-making method. Several recommendations are made based on the findings of the research study, which could be beneficial to the manufacturing sector as well as micro, small, and medium-sized businesses in Peru.

The Fourth industrial revolution has attracted much attention [12], the study findings investigate the impact of a variety of factors that have an impact on the actual adoption and implementation of Industry 4.0 in small and medium-sized enterprises in the city of Ho Chi Minh. For data collection and analysis, the researcher used a mixed-method approach, with in-depth interviews serving as one of the methods employed by the researcher. 12 participants and 396 respondents were chosen as samples by the researcher because they were representative of small and medium-sized enterprises. The primary data for this study was collected through online and offline (paper surveys). Smart PLS 3 software as well as the SPSS statistical package were used to analyze the data collected.

The industrial revolution 4.0 is already on the horizon and connected and data-driven manufacturing will have the potential to build self-optimizing facilities in which robots and computers can interact in real time [16]. The main factors influencing the adoption and implementation of Industry 4.0 for the production process of the organizations were identified, as were the problems that arose in the automated production process. This study provides a fundamental understanding of the challenges and factors influencing the implementation of Industry 4.0. The adoption of Industry 4.0 concepts by an organization helps to mitigate all its challenges. The case study method was used in this study to analyze the various issues that arise during the implementation of Industry 4.0. Sandvik Coromant was chosen as a case study for this purpose by the researchers. The data was gathered by the researcher through observations and interviews with repre- 
sentatives from five external organizations that are specialized in industrial digitalization. According to the findings of this study, there are a variety of factors that influence the adoption of Industry 4.0, which can be divided into two categories: technological factors and external environmental factors affecting the organization. According to the findings of this study, small-scale technological applications resulted in increased transparency, while increased integration of information systems resulted in a positive impact on the adoption and implementation of Industry 4.0.

Adoption of Industry 4.0 technology is a difficult challenge for SMEs due to a variety of cost and revenue restrictions [7]. There are fifteen challenges that need to be addressed before Industry 4.0 technologies can be applied in SMEs. To gather data and information from academic and industry experts, created a well-structured questionnaire. The DEMATEL approach was used by the researcher to analyze the data. This approach had been used to determine the degree of influence and interrelationship with the challenges. This research also contributes to the measurement of cause and effect in a variety of factors. The findings of the research and the results of the DEMATEL approach were validated using sensitivity analysis. Customer and partner disinterest and lack of support were found to have an impact on the application and implementation of Industry 4.0, according to the findings of this study.

Adoption and maintenance of new and innovative technologies were proving to be difficult tasks at times. The failure of Industry 4.0 technologies was a source of concern for some organizations. In addition, the research findings and results will assist small and medium-sized enterprises in formulating business and marketing strategies for implementing Industry 4.0 technologies to ensure the long-term viability of their business operations.

\section{Aims/objectives of the study}

In this study, the primary goal is to identify the various factors that have an impact on Industry 4.0 while also evaluating the impact of Industry 4.0 on the overall performance of the industrial sector. The partial goal is:

1. To identify the difficulties associated with implementing Industry 4.0 in an organization.

2. To provide various practical and theoretical implications and suggestions.

Queries for further research exploration. Two research questions have been formulated to achieve the objectives of the research study and its structure:

- RQ 1- What are the various factors that have an impact on Industry 4.0, and what are the potential challenges associated with implementing Industry 4.0 in business for the purpose of ensuring long-term development and growth of company's operations?

- RQ 2- How can the concepts of Industry 4.0 be applied to reduce the challenges that arise because of the covid 19 pandemic? 


\section{$4 \quad$ Research methodology}

An exploratory research approach is used by researchers to discover the characteristics and relationships of new research phenomena. This approach is particularly useful when researchers are looking for answers to the questions of what and how they came to be discovered. The purpose of this research study is to determine which factors have an impact on Industry 4.0, as well as how these factors should be determined. Because of the uncertainty surrounding the findings of factors, an exploratory research approach is the most appropriate method of conducting a research study. The theory and literature that are relevant to the research topic are the most important data to collect in an exploratory research approach. This paper makes use of a structured review of the available literature, with the goal of identifying the factors that are affecting the industry 4.0 revolution. The primary goal of the process was to determine whether the participants had a basic knowledge and understanding of the relevant aspects of the review. For this purpose, researchers collect relevant information and data with the help of secondary data collection sources such as published research papers, journals, peer review journals, periodicals, magazine articles, and other similar sources of information. Researchers employ a variety of keywords to search for and analyze the information they have gathered.

\subsection{Industry 4.0}

Industry 4.0 (IR4.0) is the most important issue in the current situation because the entire world is dealing with the pandemic crisis known as Covid 19, which is causing significant changes in industrial functions and the overall operation of businesses. Manufacturing, supply chain, distribution system, consumer relationship, and other aspects of business operation are all impacted by all these modifications. To achieve this, businesses must adopt new technology to perform work from employees, reach many customers, and maintain control over the supply chain and distribution system. As a result, most industries are concentrating on the adoption and implementation of Industry 4.0. Industry 4.0 is forcing organizations to adopt advanced digital technologies, which can help to minimize challenges and problems associated with technology adaptation, as well as strategic issues associated with improving the performance of the organization. However, it is necessary to implement IR4.0 in the organization.

Industry 4.0 explains how systems and objects of organizations can be connected easily and how physical information can be translated into digital format. It also explains how to analyze, communicate, and use that information to handle further intelligent action back in the physical format to complete a physical-to-digital to-physical transition.

Industry 4.0 is a term that was first used in 2011 by the German government to refer to the establishment of an initiative. It is a critical strategy for industrial production as well as the overall performance of an organization [14]. Manufacturers, producers, and related industries are undergoing a digital transformation known as Industry 4.0, which also includes value creation processes." 


\subsection{Dynamic factors impacting industry 4.0}

1. Innovation- Industry 4.0 brings about changes in production management, strategic management, and market and business strategies because of technological innovation, market, and business strategies. Innovation provides a competitive advantage and contributes to the expansion of an organization. To achieve this, organizational innovation in technology and technical skills is proposed. Innovative thinking contributes to the enhancement of organizational abilities and capabilities, which will enable organizations to adapt to the Fourth Industrial Revolution (4IR). Organizations will adopt technology-based management because it contributes to the enhancement of traditional organizational management techniques. Innovative thinking contributes to the implementation of new technologies and the transformation of business processes, and as a result, innovative thinking will have a significant impact on the performance of an organization. If an organization adopts innovation, it will have a positive impact on Industry 4.0; however, if an organization is unable to adopt innovation, it will face a variety of challenges. As a result, innovation is the most important factor influencing Industry 4.0.

2. Management- The management of the organization is critical to the successful implementation of Industry 4.0 initiatives. It was the management that had a direct impact on the choice of technology and strategic approach. It is the management of an organization that has an impact on the organizational and overall performance of the business that is being operated. In addition, managerial decisions aid in the development of skills and techniques, as well as the communication and business activities of every organization. Effective leadership, imitation participation in group work and management, as well as the control and direction of team members, all contribute to this goal. Management establishes strategic objectives, rules, regulations, and policies, and then concentrates on achieving all these objectives using innovative technologies in manufacturing, marketing, and all other aspects of the company's business operations. In any organization, managers play a critical role in the planning, decision-making and communication of the team or individuals throughout the industry. Managers are responsible for the overall success of the organizations 4.0 initiative. Management and leaders with a positive attitude adopt new technologies to minimize challenges and improve the performance of business activities. Traditional leaders, on the other hand, are reluctant to adopt new technology and to change because they are afraid of failing. All this discussion has pointed to management as being the most important factor influencing Industry 4.0 implementation.

3. Strategies - Every business organization operates and runs based on a fundamental strategy that contributes to the improvement of the efficiency and effectiveness of the organization to achieve its long- and short-term objectives and goals. Members who actively participate in the organizational environment of the organization and projects are required. All strategic decisions and strategies are influenced by the adoption of new technologies and aid in the survival of organizations in a competitive environment with uncertain conditions. Industry 4.0 focuses on the strategic aspects of an organization, which aids in gaining an understanding of the shifts occurring in the industry. Any time a change is required in a strategy for the formulation, 
planning, implementation, control, and review of new technologies and strategies adopted by an organization, a strategy change request should be submitted. The importance of Industry 4.0 in achieving organizational success is emphasized in the strategic policies of the organization in question.

4. Environment- Industry 4.0 adoption is influenced significantly by the organization's environmental factors. This is true because every organization has its own unique environment that differs from that of the other organizations. As a result, every organization operates and thrives in a distinct and dynamic environment. The term "environment" refers to all the external factors that influence the performance of organizations. It includes political, social, cultural, economic, technological, and legal factors, among others. Every organization is constantly confronted with changes in a wide range of environmental factors, all of which are complex and out of control. As a result, the organizational environment is confronted with significant challenges, the most common of which is to minimize change and achieve organizational goals in the shortest amount of time and money possible with the assistance of Industry 4.0.

5. Human resources and social determinants. - In the recent time of covid 19 pandemic, organizations must properly manage their human resources through the use of advanced and innovative technologies. Because covid 19 affected on the social life of the employee, most organizations and manufacturing were closed for a long period of time. Covid 19 gives birth to work from home trends, social distancing, travel restrictions, and other such practices, all of which are detrimental to the organization's bottom line. Industry 4.0 recognizes the importance of human involvement in all aspects of business operations.

6. Security- Organizations required technical support for each business activity during the adoption and implementation of Industry 4.0. As a result, when implementing Industry 4.0, organizations must pay particular attention to data security and ownership. If the technology that has been implemented does not provide adequate security, it will have an impact on the use of this type of technology. It was necessary for the organization to implement a high-security application to protect valuable consumer information as well as all of the data and information of the organization. Every organization should place a strong emphasis on new Industry 4.0 applications and technologies, which have the potential to reduce security risks in the same way that older systems have.

\section{$5 \quad$ Outcomes}

The findings of this study reveal that a variety of factors, including human/social factors, business environment factors, technological factors, business strategy, organizational culture, financial condition of the organization, and organizational support, among others, have an impact on the implementation of Industry 4.0.

Every organization experience changes in its organizational business process because of a variety of factors; as a result, organizations must adopt innovative and supportive technology to minimize the impact of these changes. All these changes had an 
impact on organizational culture, organizational structure, organizational strategy, management abilities, employee orientation, and other aspects of the organization. The organization then focused on implementing Industry 4.0 to facilitate innovation skills and the surrounding business environment, among other things. To ensure the successful implementation of IR4.0 at the organizational level in any industry, it is critical to incorporate innovation into management strategy, organizational environment, projectrelated issues, and human/social issues. These findings lend support to the existing literature. In general, all the factors mentioned above have a significant impact on the performance of the industry.

\section{Conclusion}

This research contributes to the understanding and analysis of all the possible factors that have an impact on the Industrial 4.0 revolution. The effective use of available data and information can aid in the formulation of strategic business objectives such as longterm growth and increased efficiency in all business operations, among other things. Internet and technology-based applications, which can be used to improve the efficiency and performance of a value chain, supply chain management, customer relationship management (CRM), and various stakeholders are driving some of this transformation. The study contributes to the realization of Industry 4.0 by requiring a clear understanding of digitalization as well as the application of Industry 4.0 to transform the physical into digital and vice versa.

This study demonstrates that the use of Industry 4.0 technologies will increase the effectiveness and efficiency of organizations and industries. This is beneficial because organizations and industries need to develop and survive in this competitive era. Adoption of smart machine and smart product applications will become more common with the widespread use of information and communication technology for all aspects of business operations and activities. This new industrial revolution incorporates these concepts and applications into business performance to maximize their efficiency and power to compete in a global competitive environment.

At the end of the study's findings, it was discovered that the development and improvement of human resource management, perceived on-time delivery, perceived cost-saving and time saving all have a positive significant effect on the usefulness of Industry 4.0. It was also discovered that digitalization and use of innovative technology in business resources, business environment, as well as ease-of-use technology all have a positive significant effect on the usefulness of Industry 4.0. The adoption objective and intention of Industry 4.0 have a significant positive impact on customer relationship management, supply chain management, and other aspects of business operations.

\section{Constraints and further orientation}

Because researchers rely on secondary sources to collect data and ascertain results, this study recommends that an interview-based survey and a well-designed questionnaire will aid in developing, formulating, and validating research plans. It will also aid 
in developing better research. Future research may be able to improve the study by considering all of its shortcomings.

Future research could increase the sample size for the survey, because a larger sample size could result in more accurate generalization of the results. One of the most effective methods of identifying the best result may be to use systematic scientific procedures to analyze the data that has been collected. It could also be beneficial in solving a problem. Finally, the research study does not consider what challenges were encountered and how they were overcome at the time of the implementation of Industry 4.0. Consequently, it could not recommend an implementation strategy to mitigate the difficulties. Therefore, future research could be focused on how to deal with the problems that are currently being faced.

\section{Theoretical implications}

There are several theoretical implications to this research study that will be useful in future research. The top management of the organization should concentrate on raising awareness about the benefits of adopting Industry 4.0 technologies. This research provides a theoretical framework for developing strategies for the adoption of innovative technologies for the advancement of sustainable and ethical development. In the manufacturing and distribution services industries, as well as in the management of human resources and customer relationships, Covid 19 creates a variety of challenges and issues. This research will provide guidance on how to develop strategic and marketing plans that will help them become more competent and capable of dealing with the entire crisis. In this context, academic experts and researchers should concentrate their various studies and research to determine future directions. The findings and observations from this research will be useful in reducing the difficulties associated with technology adoption.

\section{$9 \quad$ References}

[1] Bibby, L., \& Dehe, B. (2018). Defining and assessing industry 4.0 maturity levels - case of the defence sector. Production Planning \& Control, 29(12), 1030-1043. https://doi.org/ $10.1080 / 09537287.2018 .1503355$

[2] Bhatia, M., \& Kumar, S. (2020). Critical Success Factors of Industry 4.0 in Automotive Manufacturing Industry. IEEE Transactions on Engineering Management, 1-15. https://doi. org/10.1109/tem.2020.3017004

[3] Elbestawi, M., Centea, D., Singh, I., \& Wanyama, T. (2018). SEPT Learning Factory for Industry 4.0 Education and Applied Research. Procedia Manufacturing, 23, 249-254. https://doi.org/10.1016/j.promfg.2018.04.025

[4] Fatorachian, H., \& Kazemi, H. (2018). A critical investigation of Industry 4.0 in manufacturing: theoretical operationalization framework. Production Planning \& Control, 29(8), 633-644. https://doi.org/10.1080/09537287.2018.1424960

[5] Huang, C., Talla Chicoma, E., \& Huang, Y. (2019). Evaluating the Factors that are Affecting the Implementation of Industry 4.0 Technologies in Manufacturing MSMEs, the Case of Peru. Processes, 7(3), 161. https://doi.org/10.3390/pr7030161 
Paper-An In-Depth Analysis of the Various Factors Influencing Industry 4.0 and its Impact on the...

[6] Kiraz, A., Canpolat, O., Özkurt, C., \& Taşkın, H. (2020). Analysis of the factors affecting the industry 4.0 tendency with the structural equation model and an application. Computers \& Industrial Engineering, 150, 106911. https://doi.org/10.1016/j.cie.2020.106911

[7] Kumar, R., Singh, R., \& Dwivedi, Y. (2020). Application of industry 4.0 technologies in SMEs for ethical and sustainable operations: Analysis of challenges. Journal Of Cleaner Production, 275, 124063. https://doi.org/10.1016/j.jclepro.2020.124063

[8] Lin, B., Wu, W., \& Song, M. (2019). Industry 4.0: driving factors and impacts on firm's performance: an empirical study on China's manufacturing industry. Annals Of Operations Research. https://doi.org/10.1007/s10479-019-03433-6

[9] Lopes de Sousa Jabbour, A., Jabbour, C., Godinho Filho, M., \& Roubaud, D. (2018). Industry 4.0 and the circular economy: a proposed research agenda and original roadmap for sustainable operations. Annals Of Operations Research, 270(1-2), 273-286. https://doi.org/10. 1007/s10479-018-2772-8

[10] Lekan, A., Aigbavboa, C., Emetere, M., \& Owolabi, J. (2021). Adapting Disruptive Applications in Managing Quality Control Systems in Intelligence Manufacturing. Quality Control - Intelligent Manufacturing, Robust Design and Charts. https://doi.org/10.5772/intech open.93979

[11] Lin, D., Lee, C., Lau, H., \& Yang, Y. (2018). Strategic response to Industry 4.0: an empirical investigation on the Chinese automotive industry. Industrial Management \& Data Systems, 118(3), 589-605. https://doi.org/10.1108/imds-09-2017-0403

[12] Nguyen, X., \& Luu, Q. (2020). Factors Affecting Adoption of Industry 4.0 by Small- and Medium-Sized Enterprises: A Case in Ho Chi Minh City, Vietnam. The Journal of Asian Finance, Economics and Business, 7(6), 255-264. https://doi.org/10.13106/jafeb.2020. vol7.no6.255

[13] Nguyen, N., Thai, T., Pham, H., \& Nguyen, G. (2020). CDIO Approach in Developing Teacher Training Program to Meet Requirement of the Industrial Revolution 4.0 in Vietnam. International Journal of Emerging Technologies in Learning (Ijet), 15(18), 108. https://doi.org/10.3991/ijet.v15i18.15517

[14] Patil, K. (2021). Industry 4.0 Adoption in Manufacturing Industries Using Technology-Organization-Environment Framework. Journal Of Information Technology Research, 14(1), 123-146. https://doi.org/10.4018/jitr.2021010108

[15] Rojko, A. (2017). Industry 4.0 Concept: Background and Overview. International Journal of Interactive Mobile Technologies (Ijim), 11(5), 77. https://doi.org/10.3991/ijim.v11i5. $\underline{7072}$

[16] Winberg, E., \& Ahrén, J. (2018). Industry 4.0 from a technology adoption perspective: A case study at Sandvik Coromant (Dissertation). Retrieved from http://urn.kb.se/resolve? urn=urn:nbn:se:kth:diva-232575

[17] Yusuf, B., Walters, L., \& Sailin, S. (2020). Restructuring Educational Institutions for Growth in the Fourth Industrial Revolution (4IR): A Systematic Review. International Journal of Emerging Technologies in Learning (Ijet), 15(03), 93. https://doi.org/10.3991 /ijet.v15i03.11849

\section{Author}

Pritam Kumar has completed his bachelor's degree from Delhi University, Master of Science degree in Computer Information Systems from Assumption University, Post Graduate Diploma in IT \& Management from All India Management Association, Post Graduate Certificate in Management in Digital Marketing and Strategy from All India 
Management Association, and currently waiting for the award of Post Graduate Certificate in Management in Business Analytics from All India Management Association. In addition, he has obtained professional certifications in Business Intelligence, Business Analytics, Predictive Analytics, Marketing and Sales Analytics, HR analytics, Retail Analytics, Business Analysis and ERP. He is currently working as a lecturer in the Department of Digital Business Management, Martin de Tours School of Management and Economics, Assumption University, Bangkok, Thailand. Altogether he has 17 years of academic and five years of corporate experience. His research areas are Data Science, Digital Marketing, IR 4.0, Business Process Management, Business Analytics, Knowledge Management, and IoT.

Article submitted 2021-07-07. Resubmitted 2021-08-05. Final acceptance 2021-08-05. Final version published as submitted by the author. 